\title{
Reporter gene imaging "visualized" the integration of two growing technologies: CRISPR/Cas9-based genome editing and induced pluripotent stem cell therapy
}

\author{
Arturo Chiti ${ }^{1,2}$ \\ Published online: 19 November 2020 \\ (C) Springer-Verlag GmbH Germany, part of Springer Nature 2020
}

Cardiovascular disease is the leading cause of morbidity and mortality worldwide. However, the postmitotic human cardiomyocytes could not regenerate after injuries such as myocardial infarction (MI), resulting in adverse remodelling and ischemic cardiomyopathy, which eventually develop into heart failure. Generation of induced pluripotent stem cells (iPSCs) was first described in 2006 by Dr. Shinya Yamanaka, who received the Nobel Prize in physiology and medicine in 2012 [1]. From then on, hiPSCs have emerged as a promising source for clinical translation of cell therapy. hiPSCs can be differentiated into the three major cardiovascular cell types: cardiomyocytes, vascular smooth muscle cells and endothelial cells [2]. Numerous preclinical studies have explored the efficacy of hiPSCs derived cardiomyocytes (CMs) transplantation therapy in both small and large animal models of MI [3, 4]. More recently, Dr. Yoshiki Sawa from Osaka University in Japan performed the first in human transplantation of hiPSC-CMs, grafting sheets of 100 million cardiac cells on to the heart of a patient with ischemic cardiomyopathy. Despite the promising results from these pilot studies, several critical issues such as the optimal cell dose or delivery method, in vivo cellular retention, survival and engraftment remain unclear, limiting the therapeutic benefit and illustration of underlined mechanism [5]. Therefore, imaging methods that can monitor the in vivo behaviour of living cells could be helpful to determine essential parameters of transplantation, improving the outcome of stem cell therapy [6]. However, previous gene integration approaches rely on

This article is part of the Topical Collection on Preclinical Imaging

Arturo Chiti

arturo.chiti@hunimed.eu

1 Humanitas Clinical and Research Center - IRCCS, via Manzoni 56, 20089 Rozzano (Mi), Italy

2 Department of Biomedical Sciences, Humanitas University, Via Rita Levi Montalcini 4, 20090 Pieve Emanuele, Milan, Italy retrovirus, lentivirus, or transposon elements, which mediate random transgene integration into different loci. Random integration of gene expression components is not suitable for clinical translation due to malignant transformation associated with insertional mutagenesis [7]. For non-invasive longitudinal tracking of transplanted cells, genetic engineering is required for the constitutive expression of reporter genes in stem cells [8].

In this issue, Gao et al. investigated the dynamic of hiPSC$\mathrm{CMs}$ in the heart of MI rat model after transplantation and correlate the therapeutic effects of cell survival [9]. They genetically engineered human urinary iPSCs to express firefly luciferase (Fluc) for bioluminescence imaging (BLI), and herpes simplex virus thymidine kinase (HSVtk) for PET imaging. With the recently developed CRISPR/Cas9 mediated genome editing method, the authors were able to specifically inserted the reporter genes into the in the AAVS1 locus [10]. Thus, the resulted human cells are safe for human application because genetic modification in this "safe harbour" locus is precise. Moreover, once the transplanted cells fulfil the task or present adverse effects, the HSVtk can also serve as a "suicide gene" which allows targeted removal of transplanted cells by ganciclovir [11]. This design further increases the clinical safety of cell therapy. Coincidentally, Drs. Emmanuelle Charpentier and Jennifer Doudna were awarded the 2020 Nobel Prize in chemistry this October for developing this genome editing method. Taken together, this study utilized CRISPR/Cas9 based genome editing to generate clinical applicable transgenetic hiPSCs for the first time, allowing accurate assessment of the post-transplantation engraftment and therapeutic effects with multimodal molecular imaging.

Poor cellular engraftment and survival are the significant hurdles of stem cell therapy, especially for cell delivery into the heart [12]. The authors were able to monitor the cell fate of hUiPSC-CMs in vivo after transplantation into the MI rat heart for 5 weeks, for the first time, using a multimodal imaging technique. Their results revealed that the engrafted cells not only survived but also proliferated overtime. One critical 
question of stem cell-based cardiac therapy is the optimal cell type. Pluripotent stem cells (PSCs) derived cardiac progenitor cells is another promising cell type, which is expected to proliferate and regenerate the injured heart by giving rise to more functional muscle cells $[13,14]$. However, whether these cells can effectively differentiate into the desired cell types remains unclear [15]. Also, the potential tumorigenicity of undifferentiated cells is another safety concern before moving towards clinical study. The fate determined hPSC-CMs are considered to have limited capability of repopulating $\mathrm{CMs}$ in the tissue. Human pluripotent stem cells derived CMs have shown the great potential of proliferation and plasticity resembling the human fetal CMs $[16,17]$. The hUiPSC-CMs used in this study retain the post-transplantation mitotic capability and perfectly integrate into the host heart tissue. Beside directly regenerating the cardiac muscle tissue, the hiPSC-CMs also present impressive therapeutic effects against MI by directly secreting protective factors or via extracellular vesicles. In this case, hiPSC$\mathrm{CMs}$ are excellent cell source for cardiac regeneration therapy.

From the clinical translation prospect, outcomes of cell therapy are variable among patients due to complicated individual conditions. Thus, non-invasive imaging-based longitudinal assessment of stem cell engraftment could play an essential role in the personalized evaluation of the cell therapy and adjustments of the follow-up treatment. The CRISPR/ Cas9 based genetic engineering of hiPSCs enables the safe and straightforward integration of the clinically relevant reporter genes such as HSVtk, allowing both PET imaging and removal of the cells. The genetically engineered hiPSCCMs hold great potential in regenerative medicine for cardiovascular diseases.

\section{Compliance with ethical standards}

Conflict of interest The author declares that there are no conflicts of interest.

Ethical approval Institutional Review Board approval was not required because the paper is an Editorial.

Informed consent Not applicable.

\section{References}

1. Takahashi K, Tanabe K, Ohnuki M, Narita M, Ichisaka T, Tomoda $\mathrm{K}$, et al. Induction of pluripotent stem cells from adult human fibroblasts by defined factors. Cell. 2007;131:861-72. https://doi. org/10.1016/j.cell.2007.11.019.

2. Kattman SJ, Witty AD, Gagliardi M, Dubois NC, Niapour M, Hotta A, et al. Stage-specific optimization of activin/nodal and BMP signaling promotes cardiac differentiation of mouse and human pluripotent stem cell lines. Cell Stem Cell. 2011;8:228-40. https://doi. org/10.1016/j.stem.2010.12.008.

3. Lalit PA, Hei DJ, Raval AN, Kamp TJ. Induced pluripotent stem cells for post-myocardial infarction repair: remarkable opportunities and challenges. Circ Res. 2014;114:1328-45. https://doi.org/10. 1161/CIRCRESAHA.114.300556.

4. Oikonomopoulos A, Kitani T, Wu JC. Pluripotent stem cell-derived cardiomyocytes as a platform for cell therapy applications: Progress and hurdles for clinical translation. Mol Ther. 2018;26:1624-34. https://doi.org/10.1016/j.ymthe.2018.02.026.

5. Nguyen PK, Lan F, Wang Y, Wu JC. Imaging: guiding the clinical translation of cardiac stem cell therapy. Circ Res. 2011;109:96279. https://doi.org/10.1161/CIRCRESAHA.111.242909.

6. Di Baldassarre A, Cimetta E, Bollini S, Gaggi G, Ghinassi B. Human-induced pluripotent stem cell technology and cardiomyocyte generation: progress and clinical applications. Cells. 2018;7. https://doi.org/10.3390/cells7060048.

7. Hacein-Bey-Abina S, Von Kalle C, Schmidt M, McCormack MP, Wulffraat N, Leboulch P, et al. LMO2-associated clonal T cell proliferation in two patients after gene therapy for SCID-X1. Science. 2003;302:415-9. https://doi.org/10.1126/science.1088547.

8. Zhang JZ, Guo H, Wu JC. Applications of genetically engineered human pluripotent stem cell reporters in cardiac stem cell biology. Curr Opin Biotechnol. 2018;52:66-73. https://doi.org/10.1016/j. copbio.2018.03.002.

9. Gao Y, Wu S, Pan J, Zhang K, Li X, Xu Y, et al. CRISPR/Cas9edited triple-fusion reporter gene imaging of dynamics and function of transplanted human urinary induced pluripotent stem cellderived cardiomyocytes. Eur J Nucl Med Mol Imaging. 2020. https://doi.org/10.1007/s00259-020-05087-0.

10. Makarova KS, Haft DH, Barrangou R, Brouns SJ, Charpentier E, Horvath $\mathrm{P}$, et al. Evolution and classification of the CRISPR-Cas systems. Nat Rev Microbiol. 2011;9:467-77. https://doi.org/10. 1038/nrmicro2577.

11. Rossignoli F, Grisendi G, Spano C, Golinelli G, Recchia A, Rovesti $\mathrm{G}$, et al. Inducible Caspase9-mediated suicide gene for MSC-based cancer gene therapy. Cancer Gene Ther. 2019;26:11-6. https://doi. org/10.1038/s41417-018-0034-1.

12. Liu J, Narsinh KH, Lan F, Wang L, Nguyen PK, Hu S, et al. Early stem cell engraftment predicts late cardiac functional recovery: preclinical insights from molecular imaging. Circ Cardiovasc Imaging. 2012;5:481-90. https://doi.org/10.1161/CIRCIMAGING.111. 969329.

13. Menasche P, Vanneaux V, Hagege A, Bel A, Cholley B, Parouchev A, et al. Transplantation of human embryonic stem cell-derived cardiovascular progenitors for severe ischemic left ventricular dysfunction. J Am Coll Cardiol. 2018;71:429-38. https://doi.org/10. 1016/j.jacc.2017.11.047.

14. Kattman SJ, Huber TL, Keller GM. Multipotent flk-1+ cardiovascular progenitor cells give rise to the cardiomyocyte, endothelial, and vascular smooth muscle lineages. Dev Cell. 2006;11:723-32. https://doi.org/10.1016/j.devcel.2006.10.002.

15. Protze SI, Lee JH, Keller GM. Human pluripotent stem cell-derived cardiovascular cells: from developmental biology to therapeutic applications. Cell Stem Cell. 2019;25:311-27. https://doi.org/10. 1016/j.stem.2019.07.010.

16. Laflamme MA, Chen KY, Naumova AV, Muskheli V, Fugate JA, Dupras SK, et al. Cardiomyocytes derived from human embryonic stem cells in pro-survival factors enhance function of infarcted rat hearts. Nat Biotechnol. 2007;25:1015-24. https://doi.org/10.1038/ nbt1327.

17. Shiba Y, Gomibuchi T, Seto T, Wada Y, Ichimura H, Tanaka Y, et al. Allogeneic transplantation of iPS cell-derived cardiomyocytes regenerates primate hearts. Nature. 2016;538:388-91. https://doi. org/10.1038/nature19815.

Publisher's note Springer Nature remains neutral with regard to jurisdictional claims in published maps and institutional affiliations. 